\title{
Data Pre-processing Techniques in the Regional Emission's Load Profiles Case
}

\author{
Kewo, Angreine; Manembu, Pinrolinvic; Nielsen, Per Sieverts
}

Published in:

2019 6th International Conference on Control, Decision and Information Technologies (CoDIT)

Link to article, DOI:

10.1109/CoDIT.2019.8820303

Publication date:

2019

Document Version

Peer reviewed version

Link back to DTU Orbit

Citation (APA):

Kewo, A., Manembu, P., \& Nielsen, P. S. (2019). Data Pre-processing Techniques in the Regional Emission's Load Profiles Case. In 2019 6th International Conference on Control, Decision and Information Technologies (CoDIT) (pp. 2016-2021). IEEE. https://doi.org/10.1109/CoDIT.2019.8820303

\section{General rights}

Copyright and moral rights for the publications made accessible in the public portal are retained by the authors and/or other copyright owners and it is a condition of accessing publications that users recognise and abide by the legal requirements associated with these rights.

- Users may download and print one copy of any publication from the public portal for the purpose of private study or research.

- You may not further distribute the material or use it for any profit-making activity or commercial gain

- You may freely distribute the URL identifying the publication in the public portal 


\section{Data Pre-processing Techniques in the Regional Emission's Load Profiles Case}

\author{
Angreine Kewo \\ Management Engineering \\ Technical University of Denmark \\ Lyngby, Denmark \\ ankewo@dtu.dk
}

\author{
Pinrolinvic Manembu \\ Informatics Engineering \\ Sam Ratulangi University \\ Manado, Indonesia \\ pmanembu@unsrat.ac.id
}

\author{
Per Sieverts Nielsen \\ Management Engineering \\ Technical University of Denmark \\ Lyngby, Denmark \\ pernn@dtu.dk
}

\begin{abstract}
Different data sources, data types and platforms are involved in modelling emissions load profiles. In our case, we model emission load profiles at the regional or city level. However, we found missing values, redundancy and inconsistency in the datasets, and in most cases data preprocessing is unavoidable. Data pre-processing converts the data into a clean and tidy dataset for the subsequent modelling steps or statistical analyses. Therefore, some common techniques for data pre-processing such as cleaning, transformation, integration, reduction and some terms in data mining such as filtering and selection have been applied in our case study. We usually do the data pre-processing of moderate problems and a small amount of data using a spreadsheet application, whereas we use the programming language to do the more complex and big data size tasks. As a result, it has been found that understanding the nature of our data collection, the data flow process and the desired output comprehensively is the key for efficiency in data pre-processing. The applied techniques have helped us to provide the proper input for modelling the regional emission load profile efficiently.
\end{abstract}

Keywords- Data pre-processing, load profile, emissions, cleaning, transformation, integration, reduction, selection, modelling.

\section{INTRODUCTION}

The right to inhale clean air in our growing cities has become a central global priority. Air pollution is one of the biggest threats to air quality. Combustion of fossil fuels creates air pollution. The main pollutants come from fossil fuels' combustion in transportation, heat production and power production. Air quality can be improved by improving energy efficiency, reducing energy consumption and/or changing the energy systems to non-polluting energy sources. In our study, we focus on using the near real time information of energy consumption as a tool for increasing energy efficiency and developing emission load profiles. Moreover, the near real time information of energy use has been used to estimate how much energy can be conserved [1] in the regions or cities. Studies show that existing modelling methods can analyse energy consumption and their associated emission profile in residential and commercial sectors [2]. As a result, most studies provide policy recommendations and/or scenario tools for governments to develop more effective policies in relation to emission reduction $[3,4]$.
In practice, the data preparation, known as data preprocessing, is the key to successful modelling. In some cases, data pre-processing may take more than half of the total time spent in the modelling phase or the data mining solution [5]. It is widely used in many areas: health, transport, energy, tourism, finance, etc., and applicable to all works or studies that involve raw data or datasets. Depending on the existing data collection, a pre-processing process that consists of various tasks may be required. In this case, data preprocessing is the basic task before conducting the emissions load profile modelling phase since it can support various data sources and formats [6]. Hence, the raw data or dataset usually need pre-processing due to noise, ambiguity, redundancy, incompleteness and inconsistent data [7,8]; in some complex cases, data pre-processing is unavoidable [9]. The commonest problems in data pre-processing are caused by missing values, data corruption and outliers [8].

In our previous case of data pre-processing in modelling a regional energy load profile, we found that the different time resolutions, different units, different formats of the input data and the requirements of the tools we used in the modelling phase made the data pre-processing tasks mandatory [6].

This work is a case study based on the ClairCity project, which is an innovative project modelling air pollution and carbon emissions. The project identifies the current air emissions or pollutant concentrations by technology and citizens's activities, behavior and practices in six pilot cities or regions: Amsterdam, Bristol, Aveiro, Liguaria, Ljubljana and Sosnowiec. Consequently, the aim is to develop local-specific policy packages in which the clean-air, low-carbon, healthy future are quantified, modelled and analysed [10].

This paper focuses on the data pre-processing techniques applied in the case study to develop the regional emissions load profile. The paper is structured as follows. Section 2 evaluates the related works; Section 3 presents the selected method and framework; Section 4 discusses and evaluates the results; Section 5 concludes the paper with the research future works.

\section{RELATED WORKS}

Some methods in energy modelling that are based on data analytics such as regression methods [11], [12], machine learning techniques [13], [14] or other statistical analysis methods [26], [27], state the pre-processing method as essential. In 1980s, Pawlak has proposed the data reduction 
method by introducing the Rough set theory. It is a mathematical tool for imperfect data analysis: vague, imprecise, inconsistent and uncertain knowledge [18]-[20].

A data pre-processing method has been introduced in the power sector by focusing on data reduction, where the combination of the rough set theory and database technology is applied as an efficient solution in big data analysis. The unnecessary attributes were eliminated [19]. Studies have identified that the aim of the data reduction technique is mainly to improve efficiency. It may delete a large number of redundant data, that is why it is also called the instance selection [19], [21]-[23]. A study based on energy consumption data focuses on the missing values solution. This solution has been proposed for pre-processing by employing the interpolation technique, called Lagrange interpolation. It is a reformulation of the Newton polynomial to avoid the computation of divided differences [24].

In the data mining areas, data pre-processing tools have used specific algorithm(s) to transform the raw data into a suitable shape [26]. Subsequently, a pre-processing framework based on big data quality is introduced. It puts emphasis on the dimensions of data quality: accuracy, timeliness, consistency and completeness, and data quality rules: data domain, data types and data format. Moreover, the architecture component has covered the pre-processing sub-process including data cleansing, such as data auditing and profiling. It also covers the integration, filtering and normalisation sub-processes [27]. In analysing a complex dataset, the data pre-processing is important. Consequently, the main challenge here is to identify the most relevant subset of data in the specific classification; hence a meta-heuristic algorithm and a filter-wrapper approach were proposed in this study. The filter-wrap approach has three main stages: the discretisation, the filter and the wrapper. As a result, it shows that the proposed framework has given a higher classification accuracy and is highly reliable especially across the filter phase [9].

Validation and elimination of irrelevant data can also be introduced as a data pre-processing approach, with the aim of clustering the homogeneous behaviour [28]. It develops an extensive data pre-processing method as a phase of Knowledge Discovery from Databases (KDD) process to solve the missing values, inconsistency and some data conformance faults. Similarly, another cluster-based approach of data preprocessing has been introduced to handle a massive amount of data. It introduces a global indexing scheme that accelerates adaptive traversal of geometric data by improving the locality of hierarchical data access [29]. For this reason, a metadatadriven tool has been proposed to make the data pre-processing easier, faster and reliable, by developing the interpreter of transformation-oriented scripting [5].

\section{METHODOLOGY}

Data pre-processing is considered as one of the most important steps in data mining [23]. It will convert the data into a clean and tidy dataset for the later mining step or statistical analysis [22], [23], [29]. In statistical analysis, the four common tasks in data pre-processing are cleaning, transformation, integration and reduction [21], [22] with the aim to assess and improve the data quality for reliable statistical analysis. Cleaning, transformation, integration and reduction are the common techniques of data pre-processing in statistical analysis. The cleaning task will correct the data by filling in the missing values into a data warehouse or correct the dataset by using specific techniques, such as interpolation [24], smoothing noise data, identifying or removing outliers and resolving duplicates and inconsistencies, while minimising an introduction of bias into the data. The transformation translates, converts and/or scales the variables into the required or desired formats or units. It also covers normalisation and aggregation, where the data are transformed into the appropriate form for specific data mining algorithms purpose [22], [25].

The data integration task is utilising and synchronising multiple databases, data cubes, or files and combines these various data sources or raw datasets into one data warehouse or a single dataset. In this phase, the various datasets will be reorganised into a desired single dataset [22], [25]. The reduction task will reduce the volume and keep the same analytical results including removal of redundant records and variables [21], [22], [30]. The common problems and their solutions based on each technique are presented in the following table (Table 1).

TABLE I. DATA PRE-PROCESSING: PROBLEMS AND SOLUTION [6], [22]

\begin{tabular}{|l|l|l|}
\hline \multicolumn{1}{|c|}{ Task } & Problem/issue & \multicolumn{1}{|c|}{ Solution/Technique } \\
\hline Cleaning & Missing data & $\begin{array}{l}\text { Ignore the record } \\
\text { Determine and filling the } \\
\text { missing value manually } \\
\text { Use an expected value }\end{array}$ \\
\cline { 2 - 3 } & Noisy data & $\begin{array}{l}\text { Binning methods } \\
\text { Clustering } \\
\text { Machine learning }\end{array}$ \\
\cline { 2 - 3 } & Inconsistent data & $\begin{array}{l}\text { External reference } \\
\text { Knowledge engineering tools }\end{array}$ \\
\hline Transformation & $\begin{array}{l}\text { Different format, } \\
\text { scale or unit }\end{array}$ & Normalisation \\
\cline { 2 - 3 } & $\begin{array}{l}\text { Different standards } \\
\text { among } \\
\text { sources }\end{array}$ & $\begin{array}{l}\text { Combination data into a consistent } \\
\text { database }\end{array}$ \\
\hline Integration & $\begin{array}{l}\text { Complex analysis } \\
\text { or infeasible }\end{array}$ & $\begin{array}{l}\text { Reduce } \\
\text { observations, variables or } \\
\text { values }\end{array}$ \\
\hline & &
\end{tabular}

There are some terms, widely used in data mining area such as filtering and selection, beside the four terms mentioned before (Table 1). Reduction is also called instance selection and selection is called dimensionality reduction [19]. Data filtering uses specific criteria such as one or more attributes to filter out the data [25] and data cleansing covers data auditing and profiling [26].

\section{ANALYSIS AND RESULT}

Data pre-processing is a part of our goal to produce an emissions load profile at the regional or city level. The generic framework starts by the data collection phase, followed by the data pre-processing phase, the modelling phase and the data clustering phase. (Fig.1). 


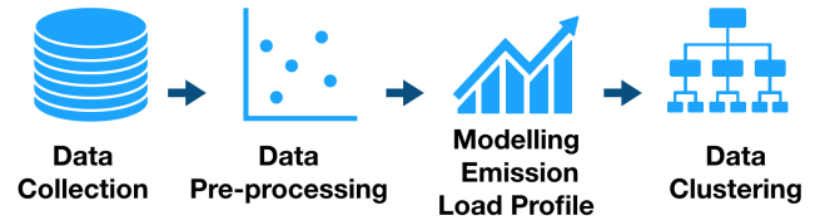

Fig. 1. Emissions load profile framework

\section{A. Data collection and selection}

The nature of different datasets in a data collection may vary. For efficiency, we evaluate the input data with the aim of identifying the data that have a significant influence on our goal to produce the emissions load profile at the regional or city level. In this case, we select the city of Sosnowiec in Poland as the case study. The nature of our collected data is a panel dataset, which is a cross sectional data sample at a specific point in time. Thus, our data collection should consist of a temperature, a national gas, a national electricity and an emissions area dataset. Furthermore, it is also essential to define the specific modelling period, in order to balance all the input data. Our period is year 2015 .

\section{B. Data pre-processing techniques}

In this section, we will present the techniques we have applied in each dataset in preparing the desired data for the modelling phase. We tend to perform the data pre-processing of moderate problems and small amount of data such as variable(s) reduction, some extraction and small amount of data scaling in a spreadsheet application. Whereas the more complex and big data size tasks, such as selection, filtering and complex calculation, we execute in Python programming language.

\section{1) Temperature:}

The temperature dataset of the case study is retrieved from a commercial weather service company. We could also have obtained it from the local meteorology office. We select the dataset from the station nearby Sosnowiec: Krakow/Balice Poland, with the coordinates: $50.08 \mathrm{~N}, 19.80 \mathrm{E}, 237 \mathrm{~m}$. The raw data are available in 365 comma-separated values (CSV) files extension, where each day represents one .CSV file. It is clear that we have to integrate the 365 files into a single dataset. Each file consists of more than 40 records, representing the half an hour temperature record but not all files have 48 records or observations. It means that the records have missing values. Each file consists of 29 variables, where we only need two variables: "datetime" and "temp". Thus, the reduction technique is required here to remove the 27 notrelevant variables. "Datetime" is in yyyymmddhhhh format and the "temp" unit is Celcius.

The first technique we apply is reduction, where we want to keep only two variables: "datetime" and "temp". Furthermore, some solutions are available online on how to merge CVS files into one single worksheet [31]. In this case, it is required to integrate the daily 365 .CSV files into one single dataset. We have applied the solution via Command Prompt (cmd), where it copies all the records from 365 files into one single file .txt file. Then, we import the txt.file into Microsoft
Excel. In this case, we have applied the transformation technique.

To overcome the missing values problem as part of the cleaning task, we have interpolated the data. Interpolation is the process of finding and constructing the unknown values based on the known values [24]. Finally, we have prepared the temperature datasets in hourly (8760 records) and daily average (365 records) resolutions to be used in the load profile modelling.

\section{2) National gas consumption pattern}

Many resources are providing the national gas consumption pattern, especially annual gas consumption data. In this case, we need the high resolution data. The higher the resolution, the better, since we want to synthesize the emissions load profile that reflects the local characteristics.

We obtain the national gas consumption pattern from Eurostat energy database [32]. The file is available in a tabdelimited (tsv) file extension. We then extract the file and open it in spreadsheet. To visualise the monthly gas consumption profile in the selected year, it is more efficient to do filtering than reduction since the file contains all the EU countries datasets in ten recent years: 2008 to 2018. It has in total 129 variables and 815 columns. The variables consists of "unit,product,indic_nrg,geo" and the rest are the time in monthly period from "2018M08" back to "2008M01". Since we have defined to model year 2015, we select "2015M01" to "2015M12". For the case study country: Poland, we select all observations that contains character "PL", because the country ID is already unique.

After we select the case study country, in the selected monthly period, we then categorise the selection based on gas unit's type, because there are two types of gas units: "MIO_M3" in million cubic metres (Fig.2) and "TJ_GCV" in Terajoules on the basis of Gross Calorific Value.

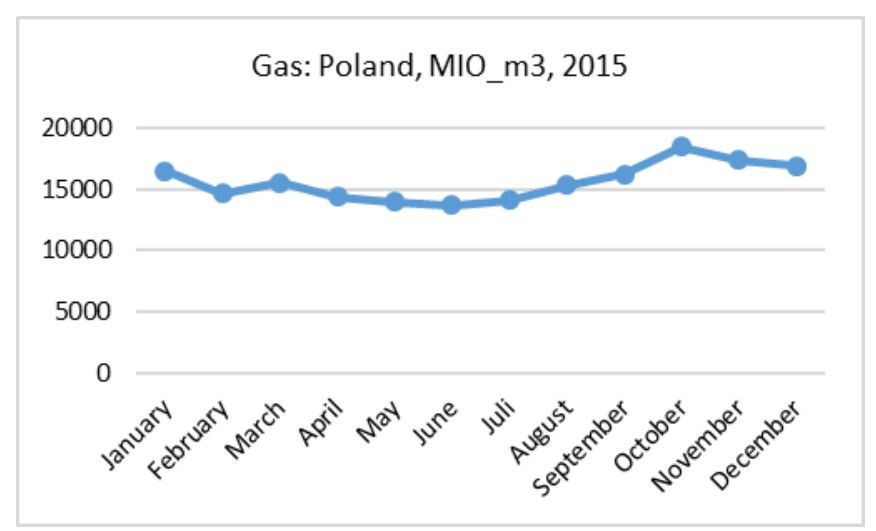

Fig. 2. Poland's monthly gas pattern, limited to unit: million meter cubic of year 2015 ( Author's calculation from [32])

Beside visualising the monthly gas consumption pattern (Fig.2), we outline the gas consumption as a monthly share, each month as a percentage of the annual consumption. (Table II). This is the final step before synthesizing the emissions load profile. 
TABLE II. POLAND'S MONTHLY GAS PATTERN AND SHARE (\%) IN 2015 (AUTHOR'S CALCULATION FROM [32])

\begin{tabular}{|l|r|r|}
\hline Month in 2015 & Unit (MIO_M3) & Gas monthly share (\%) \\
\hline January & 16475 & 8,8072404 \\
February & 14660 & 7,8369738 \\
March & 15546 & 8,3106136 \\
April & 14365 & 7,6792721 \\
May & 13957 & 7,4611626 \\
June & 13683 & 7,3146871 \\
Juli & 14086 & 7,5301237 \\
August & 15338 & 8,1994205 \\
September & 16207 & 8,6639724 \\
October & 18480 & 9,8790775 \\
November & 17377 & 9,2894335 \\
December & 16888 & 9,0280228 \\
\hline
\end{tabular}

Benchmark to other credible sources that provide monthly gas pattern is required such as benchmarking with data from Joint Organisation Data Initiative (JODI) - gas database [33] and a related gas study [34] to achieve a better synthesized result.

\section{3) National electricity consumption load}

The national electricity load dataset is available in CSV file from Open power system data [35]. It will be used as the load pattern for the commercial and the industrial sectors. The dataset has four variables:

TABLE III. NATIONAL POWER LOAD FIELD NAME AND DESCRIPTION [35]

\begin{tabular}{|l|l|l|}
\hline \multicolumn{1}{|c|}{ Fieldname } & $\begin{array}{c}\text { Type } \\
\text { (Format) }\end{array}$ & \multicolumn{1}{|c|}{ Description } \\
\hline utc_timestamp & Datetime & $\begin{array}{l}\text { Start of time period in Coordinated } \\
\text { Universal Time. }\end{array}$ \\
\hline cet_cest_timestamp & Datetime & $\begin{array}{l}\text { Start of time period in Central } \\
\text { European (Summer) Time. }\end{array}$ \\
\hline Interpolated_values & String & $\begin{array}{l}\text { Marker for missing data column in } \\
\text { source data and has been } \\
\text { interpolated. }\end{array}$ \\
\hline PL_load & Number & Total load in Poland in MW \\
\hline
\end{tabular}

The source of load value is retrieved from ENTSO-E data portal and power statistics. ENTSO-E is the European Network of Transmission System Operators. It represents 43 electricity Transmission System Operators (TSOs) from 36 countries across Europe [36]. In this case, the missing data problem has been solved by the interpolation technique from the data source.

It is required to do the reduction technique by reducing the Coordinated Universal Time variable and keeping the time stamp of Central European Time (CET) variable. We have also reduced the interpolated_values field for efficiency purpose. Therefore, the new dataset consists of two fields: CET time stamp and total load in MW, and 8760 records representing the hourly resolution.

Furthermore, it is unnecessary to perform the transformation of load value from MW to $\mathrm{kWh}$ since we only need the share of the hourly resolution in percentage. While in our previous work [6], we have performed the unit transformation to develop the regional energy load profile.
Finally, our dataset is ready to be used in the emissions load profile modelling phase.

\section{4) Residential emissions area}

The emissions area dataset is available in CSV file from our ClairCity partner. The dataset consists of eight variables: "year", "city", "zone", "codvariable", "namevariable", "pollutant", "emissions" and "unit", and 396 observations. First, we reduce some following unnecessary variables:

1. "year", is unnecessary because we have already defined model year as 2015 .

2. "city", is unnecessary because we have also defined Sosnowiec as the case city.

3. "zone" and "codvariable" are unnecessary at this moment, except we want to make further spatial analysis.

4. "unit", is unnecessary because we have agreed to used $\mathrm{Mg}$ as the emissions unit and we only need to scale the values of emissions in percentage.

Thus, we keep "namevariable", "pollutant", and "emissions" variables. "namevariable" contain the sector whether it is residential or commercial, of the combustion plants and it's fuel type. "pollutant" consist two categories, whether it is PM10 or NOX. Then "emissions" contains the value of each pollutant in the energy sector area. Furthermore, we filter and select the observations based on the energy sector categories in "namevariable": residential or commercial. Last, we quantify the total of emissions value in residential and commercial sectors per pollutant categories: NOX and PM10.

TABLE IV. SUMMARY OF REGIONAL EMISSIONS PER SECTOR (AUTHOR'S CALCULATION FROM EMISSION AREA DATASET)

\begin{tabular}{|c|c|c|c|c|}
\hline Sector & \multicolumn{2}{|c|}{ PM 10} & \multicolumn{2}{|c|}{ NOX } \\
\hline \multirow[b]{2}{*}{ Residential } & $\begin{array}{c}\text { Emissions } \\
(\mathrm{Mg})\end{array}$ & $\%$ & $\begin{array}{c}\text { Emissions } \\
(\mathrm{Mg})\end{array}$ & $\%$ \\
\hline & 1802,610 & 100,000 & 2511,290 & 100,000 \\
\hline $\begin{array}{l}\text { Residential } \\
\text { fireplaces }\end{array}$ & 0,526 & 0,029 & 0,031 & 0,001 \\
\hline Commercial & 279,326 & 100,000 & 608,955 & 100,000 \\
\hline
\end{tabular}

The emissions data description of residential and commercial sectors are presented and scaled in percentage based on each pollutant. We assume the residential fireplaces are used according to the local energy consumption characteristics when we model our emissions load profile (Table IV).

The following table has summarised all pre-processing techniques that we applied in each of our dataset. 
TABLE V. SUMMARY OF PRE-PROCESSING TECHNIQUES APPLIED IN THE CASE CITY

\begin{tabular}{|c|c|c|c|}
\hline $\begin{array}{c}\text { Data } \\
\text { collection }\end{array}$ & Property of & $\begin{array}{l}\text { Nature and type } \\
\text { of data collected }\end{array}$ & \begin{tabular}{|c|} 
Pre-processing \\
techniques
\end{tabular} \\
\hline Temperature & $\begin{array}{c}\text { Open dataset from } \\
\text { national } \\
\text { meteorology office } \\
\text { or commercial } \\
\text { weather services }\end{array}$ & $\begin{array}{c}\text { Time series } \\
\text { dataset } \\
\text { Hourly or daily } \\
\text { (average) } \\
\text { resolution }\end{array}$ & \begin{tabular}{|c|} 
Reduction \\
Integration \\
Transformation \\
Cleaning
\end{tabular} \\
\hline $\begin{array}{c}\text { Gas national } \\
\text { pattern }\end{array}$ & Eurostat & $\begin{array}{c}\text { Time series } \\
\text { dataset } \\
\text { Monthly } \\
\text { resolution }\end{array}$ & \begin{tabular}{|c|} 
Extraction \\
Filtering \\
Selection \\
Transformation \\
\end{tabular} \\
\hline $\begin{array}{c}\text { Electricity } \\
\text { national load }\end{array}$ & $\begin{array}{l}\text { Open Data } \\
\text { Platform }\end{array}$ & $\begin{array}{c}\text { Time series } \\
\text { dataset } \\
\text { Hourly } \\
\text { resolution }\end{array}$ & $\begin{array}{c}\text { Reduction } \\
\text { Transformation }\end{array}$ \\
\hline $\begin{array}{c}\text { Residential } \\
\text { emissions } \\
\text { area }\end{array}$ & $\begin{array}{l}\text { Owner: ClairCity } \\
\text { partner }\end{array}$ & Area dataset & \begin{tabular}{|c|} 
Reduction \\
Filtering \\
Selection \\
Transformation \\
\end{tabular} \\
\hline
\end{tabular}

If we compare this study with our previous work [6], this study uses a different number of data sources. Furthermore, the information we used in the data collection is different, and we employed other pre-processing techniques although we are using the same dataset. In this emissions' data pre-processing phase, we have used four kinds of data: temperature, gas, electricity and emission area. While in our previous study of energy load profile, we pre-processed seven kinds of data or information. Temperature, electricity and emissions area are the input data that be pre-processed in both of our works. For the temperature data, we have applied more data preprocessing techniques in this work than in our previous work. It is because the data structure of the raw data is different.

For the electricity data, the load of data pre-processing is quite similar as we use the same amount and type of techniques that we used previously. However, the transformation technique has been carried out in a different way, for a different purpose. In the current work, we conduct the transformation for scaling purpose, while in our previous work, the transformation is done within the unit of the data.

Last, for the emissions area dataset, it has a bigger load of data pre-processing in the current work than the previous work. In this work, we have applied four techniques, and only one technique was applied in the previous work. It is because the purpose of the data-processing is different. In this work, we aim to identify the total emission - per sector - per pollutant - in percentage and we use a specific dataset of residential fireplaces - per pollutant - in percentage. While in our previous work, we reduced two unnecessary variables and used most of the variables for spatio analysis purpose.

Based on our experience from the previous work which has employed an input dataset from a synthetic raw dataset, it could minimise the pre-processing load, since the raw data are generated using a specific algorithm. It makes no missing data issue, since a complete and consistent dataset is being generated.

\section{CONCLUSION AND RECOMMENDATION}

Understanding the nature of our data collection is important for data pre-processing efficiency [6]. We can decide the ideal technique to be used in this process. It is also fundamental to understand the process of data flow comprehensively. The moderate data pre-processing problems or some small data size problems can simply be executed in a spreadsheet application, while the more complex and big data problems may require specific programming language. Based on that, we may categorised the variable(s) reduction, some kind of extraction, small amount of data scaling and simple calculation as moderate tasks. Selection, filtering and complex calculation in big data or complicated raw data structure should be categorised as complex or hard tasks.

We agree with most of related studies that data preprocessing is a fundamental part in data analytics and that the main aim of data pre-processing is for efficiency purpose. Our study does not contribute to a new technique, but we have provided a recent and in depth case of data pre-processing in developing an emissions load profile. Finally, the selected data pre-processing techniques applied in this work have helped us to provide the proper input for modelling the regional emission load profile in a more efficient way.

A future continuation of our work could be to perform the data pre-processing analysis for spatial emissions load profile where the data structure of the input data is different than the temporal allocation load profile. It is also interesting to perform a comparison study within the ClairCity case cities or regions, since we have experienced different challenges and different nature of the input data in pre-processing each case study.

\section{ACKNOWLEDGMENT}

This work is conducted under ClairCity project funded by the European Union's Horizon 2020 research and innovation programme under grant agreement No 689289, and a $\mathrm{PhD}$ fellowship within CITIES project in Denmark Technical University (DTU) funded by Indonesia Endowment Fund for Education (LPDP). We also acknowledge ClairCity partners: PBL-The Netherlands, Techne-Italy, TML-Belgium, University of Aveiro-Portugal and all other partners for supplying the related datasets and great inputs.

\section{REFERENCES}

[1] J. Lynham, K. Nitta, T. Saijo, and N. Tarui, "Why does real-time information reduce energy consumption?," Energy Econ., vol. 54, pp. 173-181, 2016.

[2] M. G. Oladokun and I. A. Odesola, "Household energy consumption and carbon emissions for sustainable cities - A critical review of modelling approaches," Int. J. Sustain. Built Environ., vol. 4, no. 2, pp. 231-247, 2015.

[3] E. Dogan and A. Aslan, "Exploring the relationship among CO2emissions, real GDP, energy consumption and tourism in the EU and candidate countries: Evidence from panel models robust to heterogeneity and cross-sectional dependence," Renew. Sustain. Energy Rev., vol. 77, no. February, pp. 239-245, 2017.

[4] K. Ito, "CO2 emissions, renewable and non-renewable energy consumption, and economic growth: Evidence from panel data for developing countries," Int. Econ., vol. 151, no. February, pp. 1-6, 2017.

[5] P. Miksovsky, K. Matousek, and Z. Kouba, "Data pre-processing support for data mining," Syst. Man Cybern. 2002 IEEE Int. Conf., vol. 
5, p. 4 pp. vol.5, 2002.

[6] A. Kewo, P. Manembu, X. Liu, and P. S. Nielsen, "Data Pre-processing: A Case on Regional Energy Load Profile," in ICEECS 2018, 2018.

[7] B. Bilalli, A. Abelló, T. Aluja-Banet, and R. Wrembel, "Intelligent assistance for data pre-processing," Comput. Stand. Interfaces, vol. 57, no. May 2017, pp. 101-109, 2018.

[8] Z. Guo, K. Zhou, X. Zhang, S. Yang, and Z. Shao, "Data mining based framework for exploring household electricity consumption patterns: A case study in China context," J. Clean. Prod., vol. 195, pp. 773-785, 2018.

[9] S. Abdul-Rahman, A. A. Bakar, and Z. A. Mohamed-Hussein, "An intelligent data pre-processing of complex datasets," Intell. Data Anal., vol. 16, no. 2, pp. 305-325, 2012.

[10] ClairCity.eu, "ClairCity Technical Summary - Claircity.eu." [Online]. Available: http://www.claircity.eu/about/technical-summary/. [Accessed: 11-Feb-2019].

[11] A. Kewo, R. Munir, and A. K. Lapu, "IntelligEnSia based electricity consumption prediction analytics using regression method," in Proceedings - 5th International Conference on Electrical Engineering and Informatics: Bridging the Knowledge between Academic, Industry, and Community, ICEEI 2015, 2015.

[12] S. C. Yip, K. S. Wong, W. P. Hew, M. T. Gan, R. C. W. Phan, and S. W. Tan, "Detection of energy theft and defective smart meters in smart grids using linear regression," Int. J. Electr. Power Energy Syst., vol. 91, pp. 230-240, 2017.

[13] C. Robinson et al., "Machine learning approaches for estimating commercial building energy consumption," Appl. Energy, vol. 208, no. May, pp. 889-904, 2017.

[14] C. Zhang, L. Cao, and A. Romagnoli, "On the feature engineering of building energy data mining," Sustain. Cities Soc., vol. 39, no. February, pp. 508-518, 2018.

[15] M. Shahbaz, M. K. Mahalik, S. H. Shah, and J. R. Sato, "Time-varying analysis of $\mathrm{CO} 2$ emissions, energy consumption, and economic growth nexus: Statistical experience in next 11 countries," Energy Policy, vol. 98, pp. 33-48, 2016.

[16] H. Fan, I. F. MacGill, and A. B. Sproul, "Statistical analysis of drivers of residential peak electricity demand," Energy Build., vol. 141, pp. 205-217, 2017.

[17] J. An, D. Yan, and T. Hong, "Clustering and statistical analyses of airconditioning intensity and use patterns in residential buildings," Energy Build., 2018.

[18] Z. Pawlak, "Rough set theory and its applications," J. Telecommun. Inf. Technol., vol. 29, no. 7, pp. 7-10, 1998.

[19] Z. Qu and J. Liu, "A new method of power grid huge data preprocessing," Procedia Eng., vol. 15, pp. 3234-3239, 2011.

[20] Q. Zhang, Q. Xie, and G. Wang, "A survey on rough set theory and its applications," CAAI Trans. Intell. Technol., vol. 1, no. 4, pp. 323-333, 2016.

[21] G. L'Huillier and J. D. Velásquez, Advanced Techniques in Web Intelligence-2, vol. 452. 2013.

[22] MIT Critical Data, Secondary Analysis of Electronic Health Records. 2016.

[23] C. F. Tsai and J. S. Chou, "Data pre-processing by genetic algorithms for bankruptcy prediction,” IEEE Int. Conf. Ind. Eng. Eng. Manag., pp. 1780-1783, 2011.

[24] P. Manembu, A. Kewo, and B. Welang, "Missing data solution of electricity consumption based on Lagrange Interpolation case study: IntelligEnSia data monitoring," in Proceedings - 5th International Conference on Electrical Engineering and Informatics: Bridging the Knowledge between Academic, Industry, and Community, ICEEI 2015, 2015.

[25] K. J. Kim, Lecture Notes in Electrical Engineering 376 Information Science and Applications (ICISA) 2016. 2016.

[26] I. Taleb, R. Dssouli, and M. A. Serhani, "Big Data Pre-processing: A Quality Framework," Proc. - 2015 IEEE Int. Congr. Big Data, BigData Congr. 2015, pp. 191-198, 2015.

[27] F. Pinto et al., "Data Pre-processing for Database Marketing," Datagadgets, vol. 1, pp. 76-84, 2004.

[28] R. Borgo, V. Pascucci, and R. Scopigno, "Massive Data Pre-Processing with a Cluster Based Approach," Proc. 2004 Eurographics Symp. Parallel Graph. Vis., pp. 67-74, 2004.

[29] P. Aubrecht, P. Mikšovský, and L. Král, "SumatraTT: A generic data pre-processing system," Proc. - Int. Work. Database Expert Syst. Appl. DEXA, vol. 2003-Janua, pp. 120-124, 2003.

[30] F. S. Zulkepli, R. Ibrahim, and F. Saeed, "Recent Trends in Information and Communication Technology," vol. 5, 2018.

[31] Ron De Bruin, "Merge all CSV or TXT files in a folder in one worksheet," $2013 . \quad$ [Online]. Available: https://www.rondebruin.nl/win/s3/win021.htm. [Accessed: 11-Feb2019].

[32] Eurostat, "Final Energy consumption," Energy Stat. - Quant., pp. 1-6, 2010.

[33] "JODI-Gas - Data Transparency in the Global Gas Market | JODI." [Online]. Available: https://www.jodidata.org/gas/. [Accessed: 12-Feb2019].

[34] Anouk Honoré, "Natural gas demand in Europe in 2017 and short term expectations," Oxford Inst. Energy Stud., no. April, pp. 1-18, 2018.

[35] OPSD, "Data Platform - Open Power System Data." [Online]. Available: https://data.open-power-system-data.org/time_series/. [Accessed: 28-Sep-2018].

[36] "Historical data (until December 2015)." [Online]. Available: https://www.entsoe.eu/data/data-portal/. [Accessed: 28-Sep-2018]. 